\title{
Bayesian Study Using MCMC of Three-Parameter Frechet Distribution Based on Type-I Censored Data
}

\author{
Al Omari Mohammed Ahmed \\ Department of Mathematics, Faculty of Arts and Science in Qilwah, AlBaha University, Baha, KSA \\ Email: alomari1050@gmail.com
}

How to cite this paper: Ahmed, A.O.M. (2021) Bayesian Study Using MCMC of Three-Parameter Frechet Distribution Based on Type-I Censored Data. Journal of Applied Mathematics and Physics, 9, 220-232. https://doi.org/10.4236/jamp.2021.92016

Received: January 2, 2021

Accepted: January 31, 2021

Published: February 3, 2021

Copyright $\odot 2021$ by author(s) and Scientific Research Publishing Inc. This work is licensed under the Creative Commons Attribution International License (CC BY 4.0).

http://creativecommons.org/licenses/by/4.0/

\section{(c) (i) Open Access}

\begin{abstract}
Type-I censoring mechanism arises when the number of units experiencing the event is random but the total duration of the study is fixed. There are a number of mathematical approaches developed to handle this type of data. The purpose of the research was to estimate the three parameters of the Frechet distribution via the frequentist Maximum Likelihood and the Bayesian Estimators. In this paper, the maximum likelihood method (MLE) is not available of the three parameters in the closed forms; therefore, it was solved by the numerical methods. Similarly, the Bayesian estimators are implemented using Jeffreys and gamma priors with two loss functions, which are: squared error loss function and Linear Exponential Loss Function (LINEX). The parameters of the Frechet distribution via Bayesian cannot be obtained analytically and therefore Markov Chain Monte Carlo is used, where the full conditional distribution for the three parameters is obtained via Metropolis-Hastings algorithm. Comparisons of the estimators are obtained using Mean Square Errors (MSE) to determine the best estimator of the three parameters of the Frechet distribution. The results show that the Bayesian estimation under Linear Exponential Loss Function based on Type-I censored data is a better estimator for all the parameter estimates when the value of the loss parameter is positive.
\end{abstract}

\section{Keywords}

Frechet Distribution, Bayesian Method, Type-I Censored Data, Markov Chain Monte Carlo, Metropolis-Hastings Algorithm

\section{Introduction}

Frechet distribution was introduced by [1] for the largest extremes and it had 
been derived with nonnegative initial variates. Many studies have been carried out on the Frechet distribution by a lot of researchers with the aim of estimating its parameters using different statistical approaches, some of which include [2] [3] and [4]. Furthermore, [5] estimated the scale and shape parameters of Frechet distribution using principal components and least median of squares. [6] derived the reference and matching priors for the Frechet stress-strength model and developed Bayesian approach for Frechet distribution under reference prior, respectively. [7] attained Bayesian estimators of Frechet distribution and their risks by using loss functions under Gumbel Type-II prior and Levy prior. Likewise, [8] estimated the Frechet distribution parameters with an application to the medical field. As demonstrated above, no previous study dealt with Bayesian estimations of the three-parameter Frechet distribution under Type-I censored data in survival/reliability analysis.

One of the special features of survival/reliability analysis data is the censoring mechanism. There are several types of censoring mechanisms but the focus of this manuscript is in Type-I censoring. Type-I censoring is where a study is designed to end at some pre-specified given time and an event is said to have taken place if and only if the event occurs before or at the specified time period.

In this paper we want to solve the following steps:

1) To estimate the three parameters of Frechet distribution using Type I censored data by the maximum likelihood model.

2) To estimate the three parameters of Frechet distribution using Type I censored data with Jeffreys and gamma priors via Markov Chain Monte Carlo (MCMC).

3) To extend the Bayesian estimators with two loss functions, which are squared error loss function and Linear Exponential Loss Function (LINEX).

4) To assess the performance of maximum likelihood and Bayesian models through simulation study.

\section{Methodology}

\subsection{Maximum Likelihood Estimation of Frechet Censored Data}

We introduce the concept of maximum likelihood estimation on distribution with censored data. Let $\left(x_{1}, \cdots, x_{n}\right)$ be the set of $n$ random lifetime from the Frechet distribution with parameters $\lambda, \alpha$ and $\beta$. The probability density function of the Frechet distribution according to [1] is:

$$
f(x ; \lambda, \alpha, \beta)=\frac{\alpha}{\lambda}\left(\frac{x-\beta}{\lambda}\right)^{-(\alpha+1)} \exp \left(-\left(\frac{x-\beta}{\lambda}\right)^{-\alpha}\right),
$$

where the cumulative distribution function (cdf) of the Frechet distribution is given as

$$
F(x ; \lambda, \alpha)=\exp \left(-\left(\frac{x-\beta}{\lambda}\right)^{-\alpha}\right)
$$


with $\lambda$ as the scale parameter, $\alpha$ the shape parameter and $\beta$ the location parameter of the distribution

The likelihood function for a Type-I censored data as in [9] is

$$
L(\lambda, \alpha \mid x)=\prod_{i=1}^{n}\left[f\left(x_{i} ; \lambda, \alpha\right)\right]^{\delta_{i}}\left[S\left(x_{i} ; \lambda, \alpha\right)\right]^{1-\delta_{i}},
$$

where $\delta_{i}=1$ for failure and $\delta_{i}=0$ for censored observation, and $S($.$) is the$ survival function.

The logarithm of the likelihood function given in Equation (3) can be expressed as follows:

$$
\begin{aligned}
\ln L(\lambda, \alpha, \beta \mid x)= & \sum_{i=1}^{n}\left[\delta_{i}\left(\ln \alpha+\alpha \ln \lambda-(\alpha+1) \ln \left(x_{i}-\beta\right)\right)-\left(\frac{x_{i}-\beta}{\lambda}\right)^{-\alpha}\right] \\
& +\sum_{i=1}^{n}\left[\left(1-\delta_{i}\right) \ln \left(1-\exp \left(-\left(\frac{x_{i}-\beta}{\lambda}\right)^{-\alpha}\right)\right)\right]
\end{aligned}
$$

To obtain the equations for the unknown parameters, we differentiate Equation (4) partially with respect to the parameters $\lambda, \alpha$ and $\beta$ and equal it to zero. The resulting equations are given below respectively see [10],

$$
\begin{aligned}
\frac{\partial L}{\partial \lambda_{i}}= & -\frac{\alpha \sum_{i=1}^{n} \delta_{i}}{\lambda}-\frac{\alpha \sum_{i=1}^{n} \delta_{i}}{\lambda}\left(\frac{x_{i}-\beta}{\lambda}\right)^{-\alpha} \\
& +\sum_{i=1}^{n}\left(1-\delta_{i}\right)\left(\frac{\alpha\left(\frac{x_{i}-\beta}{\lambda}\right)^{-\alpha} \exp \left(-\left(\frac{x_{i}-\beta}{\lambda}\right)^{-\alpha}\right)}{\lambda\left(1-\exp \left(-\left(\frac{x_{i}-\beta}{\lambda}\right)^{-\alpha}\right)\right)}\right) \\
\frac{\partial L}{\partial \alpha_{i}}=\sum_{i=1}^{n} \delta_{i} & {\left[\frac{1}{\alpha}+\ln (\lambda)-\ln \left(x_{i}-\beta\right)+\left(\frac{x_{i}-\beta}{\lambda}\right)^{-\alpha} \ln \left(\frac{x_{i}-\beta}{\lambda}\right)\right] } \\
+\sum_{i=1}^{n} & \left(1-\delta_{i}\right)\left(\frac{\left(\frac{x_{i}-\beta}{\lambda}\right)^{-\alpha} \ln \left(\frac{x_{i}-\beta}{\lambda}\right) \exp \left(-\left(\frac{x_{i}-\beta}{\lambda}\right)^{-\alpha}\right)}{1-\exp \left(-\left(\frac{\left.\left.x_{i}-\beta\right)^{-\alpha}\right)}{\lambda}\right)\right.}\right) \\
\frac{\partial L}{\partial \beta_{i}}= & \left.\sum_{i=1}^{n} \delta_{i}\left(-\frac{\left.(\alpha-1)+\alpha\left(\frac{x_{i}-\beta}{\lambda}\right)^{-\alpha}\right)}{x_{i}-\beta}\left(-\frac{x_{i}-\beta}{\lambda}\right)^{-\alpha}\right)\right) \\
& +\sum_{i=1}^{n}\left(1-\delta_{i}\right)\left(\frac{\left(x_{i}-\beta\right)\left(1-\exp (-\beta)^{-\alpha}\right)}{\lambda\left(\frac{x_{i}-\beta}{\lambda}\right)}\right)
\end{aligned}
$$




\subsection{Bayesian Estimation of Frechet Based on Type-I Censored Data}

We consider Jeffreys prior for the scale and shape parameters. Moreover, the gamma prior will be in location parameter and the proposed prior is, see [8].

$$
\begin{gathered}
g(\lambda) \propto \frac{1}{\lambda} \\
g(\alpha) \propto \frac{1}{\alpha} \\
g(\beta / c, d) \propto \beta^{c-1} \exp (-d \beta)
\end{gathered}
$$

The joint prior distribution of three parameters is

$$
g(\lambda, \alpha, \beta / c, d) \propto \frac{1}{\lambda \alpha} \beta^{c-1} \exp (-d \beta)
$$

The posterior probability density function of the three parameters given the data $\left(x_{1}, \cdots, x_{n}\right)$ is obtained by dividing the joint probability density function over the marginal density function, following [11].

$$
\Pi(\lambda, \alpha, \beta \mid x)=\frac{L(\lambda, \alpha, \beta \mid x) g(\lambda, \alpha, \beta / c, d)}{\int_{-\infty}^{\infty} \int_{0}^{\infty} \int_{0}^{\infty} L(\lambda, \alpha, \beta \mid x) g(\lambda, \alpha, \beta / c, d) \mathrm{d} \lambda \mathrm{d} \alpha \mathrm{d} \beta}
$$

$$
=\frac{\frac{1}{\lambda \alpha} \beta^{c-1} \exp (-d \beta) \prod_{i=1}^{n}\left[\frac{\alpha}{\lambda}\left(\frac{x-\beta}{\lambda}\right)^{-(\alpha+1)} \exp \left(-\left(\frac{x-\beta}{\lambda}\right)^{-\alpha}\right)\right]^{\delta_{i}}\left[1-\exp \left(-\left(\frac{x-\beta}{\lambda}\right)^{-\alpha}\right)\right]^{1-\delta_{i}}}{\int_{-\infty}^{\infty} \int_{0}^{\infty} \int_{0}^{\infty} \frac{1}{\lambda \alpha} \beta^{c-1} \exp (-d \beta) \prod_{i=1}^{n}\left[\frac{\alpha}{\lambda}\left(\frac{x-\beta}{\lambda}\right)^{-(\alpha+1)} \exp \left(-\left(\frac{x-\beta}{\lambda}\right)^{-\alpha}\right)\right]^{\delta_{i}}\left[1-\exp \left(-\left(\frac{x-\beta}{\lambda}\right)^{-\alpha}\right)\right]^{1-\delta_{i}} \mathrm{~d} \lambda \mathrm{d} \alpha \mathrm{d} \beta}
$$

With this, the Bayesian estimates for the three parameters of Frechet distribution under squared error loss function are given as:

$$
\begin{aligned}
& \hat{\lambda}_{s}=\int_{-\infty}^{\infty} \int_{0}^{\infty} \int_{0}^{\infty} \lambda \Pi(\lambda, \alpha, \beta \mid x) \mathrm{d} \lambda \mathrm{d} \alpha \mathrm{d} \beta \\
& \hat{\alpha}_{s}=\int_{-\infty}^{\infty} \int_{0}^{\infty} \int_{0}^{\infty} \alpha \Pi(\lambda, \alpha, \beta \mid x) \mathrm{d} \lambda \mathrm{d} \alpha \mathrm{d} \beta \\
& \hat{\beta}_{s}=\int_{-\infty}^{\infty} \int_{0}^{\infty} \int_{0}^{\infty} \beta \Pi(\lambda, \alpha, \beta \mid x) \mathrm{d} \lambda \mathrm{d} \alpha \mathrm{d} \beta
\end{aligned}
$$

The Bayesian estimates under Linear Exponential Loss Function (LINEX)

$$
\begin{aligned}
& \hat{\lambda}_{l}=-\frac{1}{r} \ln \left[\int_{-\infty}^{\infty} \int_{0}^{\infty} \int_{0}^{\infty} \exp (-r \lambda) \Pi(\lambda, \alpha, \beta \mid x) \mathrm{d} \lambda \mathrm{d} \alpha \mathrm{d} \beta\right] \\
& \hat{\alpha}_{l}=-\frac{1}{r} \ln \left[\int_{-\infty}^{\infty} \int_{0}^{\infty} \int_{0}^{\infty} \exp (-r \alpha) \Pi(\lambda, \alpha, \beta \mid x) \mathrm{d} \lambda \mathrm{d} \alpha \mathrm{d} \beta\right] \\
& \hat{\beta}_{l}=-\frac{1}{r} \ln \left[\int_{-\infty}^{\infty} \int_{0}^{\infty} \int_{0}^{\infty} \exp (-r \beta) \Pi(\lambda, \alpha, \beta \mid x) \mathrm{d} \lambda \mathrm{d} \alpha \mathrm{d} \beta\right]
\end{aligned}
$$

The parameters (scale, shape and location) of the Frechet distribution cannot be solved analytically under the Bayesian approach therefore, Metropolis-Hastings Algorithm is. Used instead. 


\section{Metropolis-Hastings Algorithm}

The Metropolis-Hastings algorithm is a very general Markov Chain Mote Carlo method. It can be used to obtain random samples from any arbitrarily complicated target distribution of any dimension that is known up to a normalizing constant. In fact, Metropolis algorithm is an alternative to Gibbs sampler that does not require availability of full conditionals see [12] [13] and [14].

Therefore, the full conditional posterior density function for the three parameters given the data for Jeffreys prior with likelihood are given below

$$
\begin{aligned}
& \Pi(\lambda, \alpha, \beta \mid x) \\
& \propto \frac{1}{\lambda \alpha} \beta^{c-1} \exp (-d \beta) \prod_{i=1}^{n}\left[\frac{\alpha}{\lambda}\left(\frac{x-\beta}{\lambda}\right)^{-(\alpha+1)} \exp \left(-\left(\frac{x-\beta}{\lambda}\right)^{-\alpha}\right)\right]^{\delta_{i}} \\
& \times\left[1-\exp \left(-\left(\frac{x-\beta}{\lambda}\right)^{-\alpha}\right)\right]^{1-\delta_{i}}
\end{aligned}
$$

From Equation (20) we can get the conditional posterior of the scale parameter $\lambda$ as follows

$$
\begin{aligned}
\Pi(\lambda \mid \alpha, \beta, x) \propto & \frac{1}{\lambda} \prod_{i=1}^{n}\left[\frac{\alpha}{\lambda}\left(\frac{x-\beta}{\lambda}\right)^{-(\alpha+1)} \exp \left(-\left(\frac{x-\beta}{\lambda}\right)^{-\alpha}\right)\right]^{\delta_{i}} \\
& \times\left[1-\exp \left(-\left(\frac{x-\beta}{\lambda}\right)^{-\alpha}\right)\right]^{1-\delta_{i}}
\end{aligned}
$$

where the others the scale parameter will be constant for the conditional posterior of the scale parameter then from the posterior we can remove and get the final formula as given in Equation (21).

The conditional posterior of the shape parameter is given below

$$
\begin{aligned}
\Pi(\beta \mid \lambda, \alpha, x) \propto & \beta^{c-1} \prod_{i=1}^{n}\left[\frac{\alpha}{\lambda}\left(\frac{x-\beta}{\lambda}\right)^{-(\alpha+1)} \exp \left(-\left(\frac{x-\beta}{\lambda}\right)^{-\alpha}\right)\right]^{\delta_{i}} \\
& \times\left[1-\exp \left(-\left(\frac{x-\beta}{\lambda}\right)^{-\alpha}\right)\right]^{1-\delta_{i}}
\end{aligned}
$$

The conditional posterior of the location parameter is given below

$$
\begin{aligned}
\Pi(\alpha \mid \lambda, \beta, x) \propto & \frac{1}{\alpha} \prod_{i=1}^{n}\left[\frac{\alpha}{\lambda}\left(\frac{x-\beta}{\lambda}\right)^{-(\alpha+1)} \exp \left(-\left(\frac{x-\beta}{\lambda}\right)^{-\alpha}\right)\right]^{\delta_{i}} \\
& \times\left[1-\exp \left(-\left(\frac{x-\beta}{\lambda}\right)^{-\alpha}\right)\right]^{1-\delta_{i}}
\end{aligned}
$$

As shown in the conditional posterior of the three parameters, they do not follow any close form, hence the use of Metropolis-Hastings algorithm as detailed below to generate MCMC sample, see [15] and [16].

\section{Algorithm:}

1) Start with initial value $\lambda_{0}, \alpha_{0}, \beta_{0}$ 
2) The current value $\lambda_{i}, \alpha_{i}, \beta_{i}$ and generate the candidate value $\lambda^{*}, \alpha^{*}, \beta^{*}$ from arbitrary distribution Uniform $(0,1)$.

3) Taken the ratio at the candidate value $\lambda^{*}$ and current value $\lambda_{i}$

$$
\gamma_{1}=\min \left\{1, \frac{\Pi\left(\lambda^{*} \mid \alpha, \beta, x\right)}{\Pi\left(\lambda_{i} \mid \alpha, \beta, x\right)}\right\}
$$

The next value of $\lambda_{i}$ is given below as

$$
\lambda_{i+1}= \begin{cases}\lambda^{*} & \text { with probability } p \\ \lambda_{i} & \text { with probability } 1-p\end{cases}
$$

4) Generate $u$ from Uniform $(0,1)$ and accept $\lambda^{*}$ with probability $p$ if $\lambda^{*}<p$ and return to step 2 , otherwise accept $\lambda_{i}$ and return to step 2 .

5) Taken the ratio at the candidate value $\alpha^{*}$ and current value $\alpha_{i}$

$$
\gamma_{2}=\min \left\{1, \frac{\Pi\left(\alpha^{*} \mid \lambda, \beta, x\right)}{\Pi\left(\alpha_{i} \mid \lambda, \beta, x\right)}\right\}
$$

The next value of $\alpha_{i}$ is given below as

$$
\alpha_{i+1}= \begin{cases}\alpha^{*} & \text { with probability } p \\ \alpha_{i} & \text { with probability } 1-p\end{cases}
$$

6) Generate $u$ from Uniform $(0,1)$ and accept $\alpha^{*}$ with probability $p$ if $\alpha^{*}<p$ and return to step 2, otherwise accept $\alpha_{i}$ and return to step 2 .

7) Taken the ratio at the candidate value $\beta^{*}$ and current value $\beta_{i}$

$$
\gamma_{3}=\min \left\{1, \frac{\Pi\left(\beta^{*} \mid \lambda, \alpha, x\right)}{\Pi\left(\beta_{i} \mid \lambda, \alpha, x\right)}\right\}
$$

The next value of $\alpha_{i}$ is given below as

$$
\beta_{i+1}= \begin{cases}\beta^{*} & \text { with probability } p \\ \beta_{i} & \text { with probability } 1-p\end{cases}
$$

8) Generate $u$ from Uniform $(0,1)$ and accept $\beta^{*}$ with probability $p$ if $\beta^{*}<p$ and return to step 2 , otherwise accept $\beta_{i}$ and return to step 2 .

9) The Bayesian estimation of the scale, shape and location parameters under the squared error loss function is given as

$$
\begin{aligned}
& \hat{E}_{S}(\lambda \mid \alpha, \beta, x)=\frac{1}{n} \sum_{i=1}^{n} \lambda_{i} \\
& \hat{E}_{S}(\alpha \mid \lambda, \beta, x)=\frac{1}{n} \sum_{i=1}^{n} \alpha_{i} \\
& \hat{E}_{S}(\beta \mid \lambda, \alpha, x)=\frac{1}{n} \sum_{i=1}^{n} \beta_{i}
\end{aligned}
$$

10) The Bayesian estimation of the scale, shape and location parameters under the Linex loss function is given as

$$
\hat{E}_{L}(\lambda \mid \alpha, \beta, x)=-\frac{1}{r} \ln \left[\frac{1}{n} \sum_{i=1}^{n} \exp \left(-r \lambda_{i}\right)\right]
$$




$$
\begin{aligned}
& \hat{E}_{L}(\alpha \mid \lambda, \beta, x)=-\frac{1}{r} \ln \left[\frac{1}{n} \sum_{i=1}^{n} \exp \left(-r \alpha_{i}\right)\right] \\
& \hat{E}_{L}(\beta \mid \lambda, \alpha, x)=-\frac{1}{r} \ln \left[\frac{1}{n} \sum_{i=1}^{n} \exp \left(-r \beta_{i}\right)\right]
\end{aligned}
$$

\section{Simulation Study}

The values specified for the parameters, sample sizes, censoring time and the Linex loss function were meant for illustration purposes only and were therefore not restrictive. However, to assess the performance of the Maximum likelihood and Bayesian estimations based on Type-I censored data to estimate the three parameters of Frechet distribution, the Mean Squared Errors (MSE) were calculated after running 10,000 iterations. Different sets of sample sizes as well as censoring mechanisms were considered. The samples were $n=25,50$ and 100 and that of the censoring time was $20 \%$. The values considered for the Frechet distribution parameters were the scale parameter which was (1 and 2), the shape parameter which was $(0.4$ and 1.4$)$ and the location parameter which was 3 . Two values (both decreasing and increasing) were specified for the Linex loss function; these were; $r=-0.7$ and 0.7. The maximum likelihood (MLE) estimated the three parameters of Frechet distribution by using the numerical method with $\mathrm{R}$ programme in the Equation (4) via (maxLik) package. In the Bayesian estimation, the Metropolis-Hastings Algorithm was used in Algorithm via (pscl) package in $\mathrm{R}$ programme for Bayesian under square error loss function and LINEX loss function to estimate the three parameters of Frechet distribution, where hyper-parameters of gamma priors were equal to 1 .

The results were displayed in Tables 1-6 and indicated the different choices of the three parameters, sample size, loss parameter and censoring rate.

\begin{tabular}{|c|c|c|c|c|c|}
\hline \multirow{3}{*}{ Size } & \multirow{3}{*}{ Estimators } & \multicolumn{4}{|c|}{$\beta=3$} \\
\hline & & \multicolumn{2}{|c|}{$\lambda=1$} & \multicolumn{2}{|c|}{$\lambda=2$} \\
\hline & & $\alpha=0.4$ & $\alpha=1.4$ & $\alpha=0.4$ & $\alpha=1.4$ \\
\hline \multirow{4}{*}{25} & MLE & 1.1042 & 0.9623 & 1.9827 & 2.1314 \\
\hline & BS & 1.1486 & 0.9583 & 1.9644 & 2.1982 \\
\hline & $\mathrm{BL}(r=-0.7)$ & 1.1580 & 0.9611 & 1.9761 & 2.2124 \\
\hline & $\mathrm{BL}(r=0.7)$ & 1.0716 & 0.9858 & 1.9996 & 2.0824 \\
\hline \multirow{4}{*}{50} & MLE & 1.1062 & 0.9675 & 1.9547 & 2.1241 \\
\hline & BS & 1.1304 & 0.9493 & 1.9342 & 2.1638 \\
\hline & $\mathrm{BL}(r=-0.7)$ & 1.1354 & 0.9538 & 1.9403 & 2.1722 \\
\hline & $\mathrm{BL}(r=0.7)$ & 1.0878 & 0.9810 & 1.9822 & 2.0938 \\
\hline \multirow{4}{*}{100} & MLE & 1.1010 & 0.9495 & 1.9279 & 2.1184 \\
\hline & BS & 1.1128 & 0.9301 & 1.9122 & 2.1376 \\
\hline & $\mathrm{BL}(r=-0.7)$ & 1.1152 & 0.9321 & 1.9151 & 2.1415 \\
\hline & $\mathrm{BL}(r=0.7)$ & 1.0918 & 0.9614 & 1.9467 & 2.1034 \\
\hline
\end{tabular}

Table 1. Estimate the scale parameter of Frechet based on Type-I censored data. 
Table 2. MSE for the scale parameter of Frechet based Type-I censored data.

\begin{tabular}{|c|c|c|c|c|c|}
\hline \multirow{3}{*}{ Size } & \multirow{3}{*}{ Estimators } & \multicolumn{4}{|c|}{$\beta=3$} \\
\hline & & \multicolumn{2}{|c|}{$\lambda=1$} & \multicolumn{2}{|c|}{$\lambda=2$} \\
\hline & & $\alpha=0.4$ & $\alpha=1.4$ & $\alpha=0.4$ & $\alpha=1.4$ \\
\hline \multirow{4}{*}{25} & MLE & 0.1603 & 0.1429 & 0.1853 & 0.2014 \\
\hline & BS & 0.1766 & 0.1436 & 0.1864 & 0.2305 \\
\hline & $\mathrm{BL}(r=-0.7)$ & 0.1807 & 0.1428 & 0.1876 & 0.2379 \\
\hline & $\mathrm{BL}(r=0.7)$ & 0.1511 & 0.1419 & 0.1846 & 0.1864 \\
\hline \multirow{4}{*}{50} & MLE & 0.1392 & 0.1286 & 0.1479 & 0.1639 \\
\hline & BS & 0.1452 & 0.1293 & 0.1496 & 0.1746 \\
\hline & $\mathrm{BL}(r=-0.7)$ & 0.1463 & 0.1296 & 0.1497 & 0.1774 \\
\hline & $\mathrm{BL}(r=0.7)$ & 0.1351 & 0.1263 & 0.1463 & 0.1572 \\
\hline \multirow{4}{*}{100} & MLE & 0.1288 & 0.1219 & 0.1348 & 0.1442 \\
\hline & BS & 0.1312 & 0.1237 & 0.1358 & 0.1486 \\
\hline & $\mathrm{BL}(r=-0.7)$ & 0.1317 & 0.1245 & 0.1364 & 0.1496 \\
\hline & $\mathrm{BL}(r=0.7)$ & 0.1272 & 0.1208 & 0.1337 & 0.1412 \\
\hline
\end{tabular}

Table 3. Estimate the shape parameter of Frechet based Type-I censored data.

\begin{tabular}{|c|c|c|c|c|c|}
\hline \multirow{3}{*}{ Size } & \multirow{3}{*}{ Estimators } & \multicolumn{4}{|c|}{$\beta=3$} \\
\hline & & \multicolumn{2}{|c|}{$\lambda=1$} & \multicolumn{2}{|c|}{$\lambda=2$} \\
\hline & & $\alpha=0.4$ & $\alpha=1.4$ & $\alpha=0.4$ & $\alpha=1.4$ \\
\hline \multirow{4}{*}{25} & MLE & 0.5432 & 1.2733 & 0.4227 & 1.5116 \\
\hline & BS & 0.5876 & 1.2693 & 0.4044 & 1.5784 \\
\hline & $\mathrm{BL}(r=-0.7)$ & 0.5971 & 1.2722 & 0.4167 & 1.5926 \\
\hline & $\mathrm{BL}(r=0.7)$ & 0.5106 & 1.2968 & 0.4396 & 1.4626 \\
\hline \multirow{4}{*}{50} & MLE & 0.5452 & 1.2785 & 0.3947 & 1.5042 \\
\hline & BS & 0.5694 & 1.2603 & 0.3742 & 1.5447 \\
\hline & $\mathrm{BL}(r=-0.7)$ & 0.5744 & 1.2648 & 0.3803 & 1.5522 \\
\hline & $\mathrm{BL}(r=0.7)$ & 0.5268 & 1.2922 & 0.4222 & 1.4747 \\
\hline \multirow{4}{*}{100} & MLE & 0.5401 & 1.2605 & 0.3679 & 1.4986 \\
\hline & BS & 0.5518 & 1.2416 & 0.3522 & 1.5178 \\
\hline & $\mathrm{BL}(r=-0.7)$ & 0.5542 & 1.2431 & 0.3551 & 1.5217 \\
\hline & $\mathrm{BL}(r=0.7)$ & 0.5308 & 1.2724 & 0.3867 & 1.4836 \\
\hline
\end{tabular}


Table 4. MSE for the shape parameter of Frechet based Type-I censored data.

\begin{tabular}{|c|c|c|c|c|c|}
\hline \multirow{3}{*}{ Size } & \multirow{3}{*}{ Estimators } & \multicolumn{4}{|c|}{$\beta=3$} \\
\hline & & \multicolumn{2}{|c|}{$\lambda=1$} & \multicolumn{2}{|c|}{$\lambda=2$} \\
\hline & & $\alpha=0.4$ & $\alpha=1.4$ & $\alpha=0.4$ & $\alpha=1.4$ \\
\hline \multirow{4}{*}{25} & MLE & 0.8724 & 0.8552 & 0.8974 & 0.9135 \\
\hline & BS & 0.8887 & 0.8557 & 0.8985 & 0.9426 \\
\hline & $\mathrm{BL}(r=-0.7)$ & 0.8928 & 0.8551 & 0.8997 & 0.9501 \\
\hline & $\mathrm{BL}(r=0.7)$ & 0.8631 & 0.8543 & 0.8967 & 0.8981 \\
\hline \multirow{4}{*}{50} & MLE & 0.8511 & 0.8407 & 0.8686 & 0.8762 \\
\hline & BS & 0.8571 & 0.8411 & 0.8617 & 0.8867 \\
\hline & $\mathrm{BL}(r=-0.7)$ & 0.8584 & 0.8417 & 0.8618 & 0.8891 \\
\hline & $\mathrm{BL}(r=0.7)$ & 0.8472 & 0.8384 & 0.8584 & 0.8693 \\
\hline \multirow{4}{*}{100} & MLE & 0.8409 & 0.8343 & 0.8469 & 0.8563 \\
\hline & BS & 0.8433 & 0.8358 & 0.8479 & 0.8607 \\
\hline & $\mathrm{BL}(r=-0.7)$ & 0.8438 & 0.8366 & 0.8485 & 0.8617 \\
\hline & $\mathrm{BL}(r=0.7)$ & 0.8391 & 0.8329 & 0.8458 & 0.8533 \\
\hline
\end{tabular}

Table 5. Estimate the location parameter of Frechet based Type-I censored data.

\begin{tabular}{|c|c|c|c|c|c|}
\hline \multirow{3}{*}{ Size } & \multirow{3}{*}{ Estimators } & \multicolumn{4}{|c|}{$\beta=3$} \\
\hline & & \multicolumn{2}{|c|}{$\lambda=1$} & \multicolumn{2}{|c|}{$\lambda=2$} \\
\hline & & $\alpha=0.4$ & $\alpha=1.4$ & $\alpha=0.4$ & $\alpha=1.4$ \\
\hline \multirow{4}{*}{25} & MLE & 3.1165 & 2.9746 & 3.0061 & 3.1548 \\
\hline & BS & 3.1609 & 2.9706 & 2.9878 & 3.2216 \\
\hline & $\mathrm{BL}(r=-0.7)$ & 3.1703 & 2.9733 & 2.9994 & 3.2358 \\
\hline & $\mathrm{BL}(r=0.7)$ & 3.0839 & 2.9981 & 3.0231 & 3.1058 \\
\hline \multirow{4}{*}{50} & MLE & 3.1185 & 2.9798 & 2.9781 & 3.1474 \\
\hline & BS & 3.1427 & 2.9616 & 2.9576 & 3.1872 \\
\hline & $\mathrm{BL}(r=-0.7)$ & 3.1477 & 2.9661 & 2.9637 & 3.1954 \\
\hline & $\mathrm{BL}(r=0.7)$ & 3.1001 & 2.9933 & 3.0056 & 3.1172 \\
\hline \multirow{4}{*}{100} & MLE & 3.1133 & 2.9618 & 2.9513 & 3.1418 \\
\hline & BS & 3.1251 & 2.9423 & 2.9356 & 3.1612 \\
\hline & $\mathrm{BL}(r=-0.7)$ & 3.1275 & 2.9444 & 2.9385 & 3.1649 \\
\hline & $\mathrm{BL}(r=0.7)$ & 3.1041 & 2.9737 & 2.9701 & 3.1268 \\
\hline
\end{tabular}


Table 6. MSE for the location parameter of Frechet based Type-I censored dat.

\begin{tabular}{|c|c|c|c|c|c|}
\hline \multirow{3}{*}{ Size } & \multirow{3}{*}{ Estimators } & \multicolumn{4}{|c|}{$\beta=3$} \\
\hline & & \multicolumn{2}{|c|}{$\lambda=1$} & \multicolumn{2}{|c|}{$\lambda=2$} \\
\hline & & $\alpha=0.4$ & $\alpha=1.4$ & $\alpha=0.4$ & $\alpha=1.4$ \\
\hline \multirow{4}{*}{25} & MLE & 0.3102 & 0.2928 & 0.3352 & 0.3513 \\
\hline & BS & 0.3265 & 0.2935 & 0.3363 & 0.3804 \\
\hline & $\mathrm{BL}(r=-0.7)$ & 0.3306 & 0.2928 & 0.3375 & 0.3878 \\
\hline & $\mathrm{BL}(r=0.7)$ & 0.3009 & 0.2918 & 0.3345 & 0.3359 \\
\hline \multirow{4}{*}{50} & MLE & 0.2889 & 0.2785 & 0.2978 & 0.3138 \\
\hline & BS & 0.2949 & 0.2789 & 0.2995 & 0.3245 \\
\hline & $\mathrm{BL}(r=-0.7)$ & 0.2962 & 0.2795 & 0.2996 & 0.3269 \\
\hline & $\mathrm{BL}(r=0.7)$ & 0.2852 & 0.2762 & 0.2962 & 0.3071 \\
\hline \multirow{4}{*}{100} & MLE & 0.2787 & 0.2718 & 0.2847 & 0.2941 \\
\hline & BS & 0.2811 & 0.2736 & 0.2857 & 0.2985 \\
\hline & $\mathrm{BL}(r=-0.7)$ & 0.2816 & 0.2744 & 0.2863 & 0.2995 \\
\hline & $\mathrm{BL}(r=0.7)$ & 0.2769 & 0.2707 & 0.2836 & 0.2911 \\
\hline
\end{tabular}

\section{Results and Discussion}

Four values of the estimators which are Maximum likelihood estimation (MLE), Bayesian under squared error loss function (BS), Bayesian under Linear Exponential Loss Function with $r=-0.7$ (BL $(r=-0.7))$ and Bayesian under Linear Exponential Loss Function with $r=0.7$ (BL $(r=0.7))$ are shown in each column for each size.

Table 1 contains the estimate of the scale parameter of Frechet distribution based on Type-I censored data with Maximum likelihood, Bayesian under squared error loss function and under Linear Exponential Loss Function with $r$ $=-0.7$ and 0.7. As shown in the results when the shape paramemter $\lambda$ was 1 and location pramster $\alpha$ was 0.4 with size 25, the Maximum Likelihood Estimation (MLE) was 1.1042, the Bayesian under squared error loss function (BS) estimation was 1.1486 , BL $(r=-0.7)$ estimation was 1.1580 and BL $(r=0.7)$ estimation was 1.0716, we observed that the $\mathrm{BL}(r=0.7)$ was closer to true value than others estimation.

From Table 2 the results show that, the Bayesian estimation under Linear Exponential Loss Function with $r=0.7$ is better compared to the others with respect to the MSE of scale parameter. We observed from Table 2 that the maximum likelihood estimation has the smallest MSE and therefore is a better estimate that Bayesian under squared error loss function as well as Bayesian under Linear Exponential Loss Function with $r=-0.7$ for all cases except with size 25 when.

Table 3 presented the estimates of the shape parameter of Frechet distribution based on Type-I censored data by employing the four estimators above as illu- 
strated earlier. The initial values of the shape parameter were 0.4 and 1.4.

As shown in the results, the Maximum Likelihood Estimation (MLE) was 0.5432 and the Bayesian under squared error loss function (BS) estimation was 0.5876 . When the true value of the shape parameter was 0.4 , we observed that the MLE was closer to true value than BS estimation.

As shown in the results when the shape paramemter $\lambda$ was 1 and location pramster $\alpha$ was 0.4 with size 25, the Maximum Likelihood Estimation (MLE) was 0 5432, the Bayesian under squared error loss function (BS) estimation was 0.5876 , BL $(r=-0.7)$ estimation was 0.5971 and BL $(r=0.7)$ estimation was 0.5106 , we observed that the $\mathrm{BL}(r=0.7)$ was closer to true value than others estimation.

As shown in Table 4 the performance of the estimators for the estimation of the shape parameter was compared again using mean squared error (MSE). We again observed that the Bayesian estimation under Linear Exponential Loss Function with $r=0.7$ performed better compared to the others with respect to the MSE of scale parameter. Moreover, the maximum likelihood estimation is better than Bayesian under squared error loss function and Bayesian under Linear Exponential Loss Function with $r=-0.7$ for all cases except with size 25 when also with size 50 when Table 5 presented the estimates of the location parameter of Frechet distribution based on Type-I censored data by employing the four estimators. The initial value of the location parameter was 3. The result showed that the MLE was 3.1165 and Bayesian under Linear Exponential Loss Function with $r=-0.7$ (BL $(r=0.7)$ ) estimation was 3.0839. We observed that the Bayesian under Linear Exponential Loss Function with $r=-0.7$ (BL $(r=0.7)$ ) estimation was closer to true value than the MLE.

In Table 6 the estimators of the location parameter were compared by mean square error (MSE) and it was observed that the Bayesian estimation under Linear Exponential Loss Function with $\mathrm{r}=0.7$ is better compared to the others with respect to the MSE of scale parameter. Moreover, the maximum likelihood estimation is better than Bayesian under squared error loss function and Bayesian under Linear Exponential Loss Function with $r=-0.7$.

\section{Conclusion}

The results from this study show that the Bayesian estimation under Linear Exponential Loss Function with $r=0.7$ based on Type-I censored data was a better estimator for all the parameters (scale, shape and location) estimates. The contribution to knowledge in this manuscript was the application of the Bayesian estimation approaches based on Type-I censored data that was considered under Markov Chain Monte Carlo (MCMC) for the three parameters of Frechet distribution. The Maximum Likelihood Estimation (MLE) was better than Bayesian under square error loss function and Exponential Loss Function with $r$ $=-0.7$ for all the parameters (scale, shape and location) estimates. When the number of sample size increases, the mean squared errors (MSE) decrease in all cases. 


\section{Conflicts of Interest}

The author declares no conflicts of interest regarding the publication of this paper.

\section{References}

[1] Frechet, M. (1928) Sur la loi de probabilite de lecart maximum. Annales Polonici Mathematici, 6, 93-116.

[2] Gumbel, E.J. (1965) A Quick Estimation of the Parameters in Frechet's Distribution. Review of the International Statistical Institute, 33, 349-363. https://doi.org/10.2307/1401700

[3] Mann, R.N. (1984) Statistical Estimation of Parameters of the Weibull and Frechet Distribution. In: de Oliveira, J.T., Ed., Statistical Extremes and Applications, Vol. 131, Springer, Dordrecht, 81-89. https://doi.org/10.1007/978-94-017-3069-3 6

[4] Hooda, B.K., Singh, N.P. and Singh, U. (1993) On Estimation in Fre'chet Distribution of m-th Maxima. Communications in Statistics-Theory and Methods, 22, 907-919. https://doi.org/10.1080/03610929308831064

[5] Afify, E.D. (2004) On the Confidence Interval of the Frechet Distribution. Far East Journal of Theoretical Statistics, 12, 221-226.

[6] Abbas, K. and Tang, Y. (2015) Analysis of Frechet Distribution Using Reference Priors. Communications in Statistics-Theory and Methods, 44, 2945-2956. https://doi.org/10.1080/03610926.2013.802351

[7] Nasir, W. and Aslam, M. (2015) Bayes Approach to Study Shape Parameter of Frechet Distribution. International Journal of Basic and Applied Sciences, 4, 246-254. https://doi.org/10.14419/ijbas.v4i3.4644

[8] Abbas, K., Abbasi, N.Y., Ali, A., Khan, S.A., Manzoor, S., Khalil, A., Khalil, U., Khan, D.M., Hussain, Z. and Altaf, M. (2019) Bayesian Analysis of Three-Parameter Frechet Distribution with Medical Applications. Computational and Mathematical Methods in Medicine, 2019, Article ID: 9089856. https://doi.org/10.1155/2019/9089856

[9] Klein, J.P. and Moeschberger, M.L. (2003) Survival Analysis: Techniques for Censored and Truncated Data. Springer, New York. https://doi.org/10.1007/b97377

[10] Al Eibood, F. and Eidous, O. (2017) A Weighted Exponential Model for Grouped Line Transect Data. Mathematics and Statistics, 5, 1-4. https://doi.org/10.13189/ms.2017.050101

[11] Al Omari, M.A., Ibrahim, N.A., Arasan, J. and Adam, M.B. (2012) Bayesian Survival and Hazard Estimate for Weibull Censored Time Distribution. Journal of Applied Sciences, 12, 1313-1317. https://dx.doi.org/10.3923/jas.2012.1313.1317

[12] Hastings, W.K. (1970) Monte Carlo Sampling Methods Using Markov Chains and Their Applications. Biometrika, 57, 97-109. https://doi.org/10.1093/biomet/57.1.97

[13] Soliman, A.A., Abd-Ellah, A.H., Abou-Elheggag, N.A. and Ahmed, E.A. (2011) Modified Weibull Model: A Bayes Study Using MCMC Approach Based on Progressive Censoring Data. Reliability Engineering \& System Safety, 100, 48-57. https://doi.org/10.1016/j.ress.2011.12.013

[14] Suparman (2020) Bayesian Estimation in Piecewise Constant Model with Gamma Noise by Using Reversible Jump MCMC. Mathematics and Statistics, 8, 17-22. https://doi.org/10.13189/ms.2020.081303

[15] Al Omari, M.A. (2014) Comparison of the Bayesian Methods on Interval-Censored 
Data for Weibull Distribution. Open Journal of Statistics, 4, 570-577.

http://dx.doi.org/10.4236/ojs.2014.48053

[16] Al Omari, M.A. (2016) Bayesian Study Using MCMC of Gompertz Distribution Based on Interval Censored Data with Three Loss Functions. Journal of Applied Sciences, 16, 88-97. https://dx.doi.org/10.3923/jas.2016.88.97 